\title{
A Cylindrical Ring Dielectric Resonator based Passive Wireless Sensor for Position Insensitive Crack Monitoring \\ --Manuscript Draft--
}

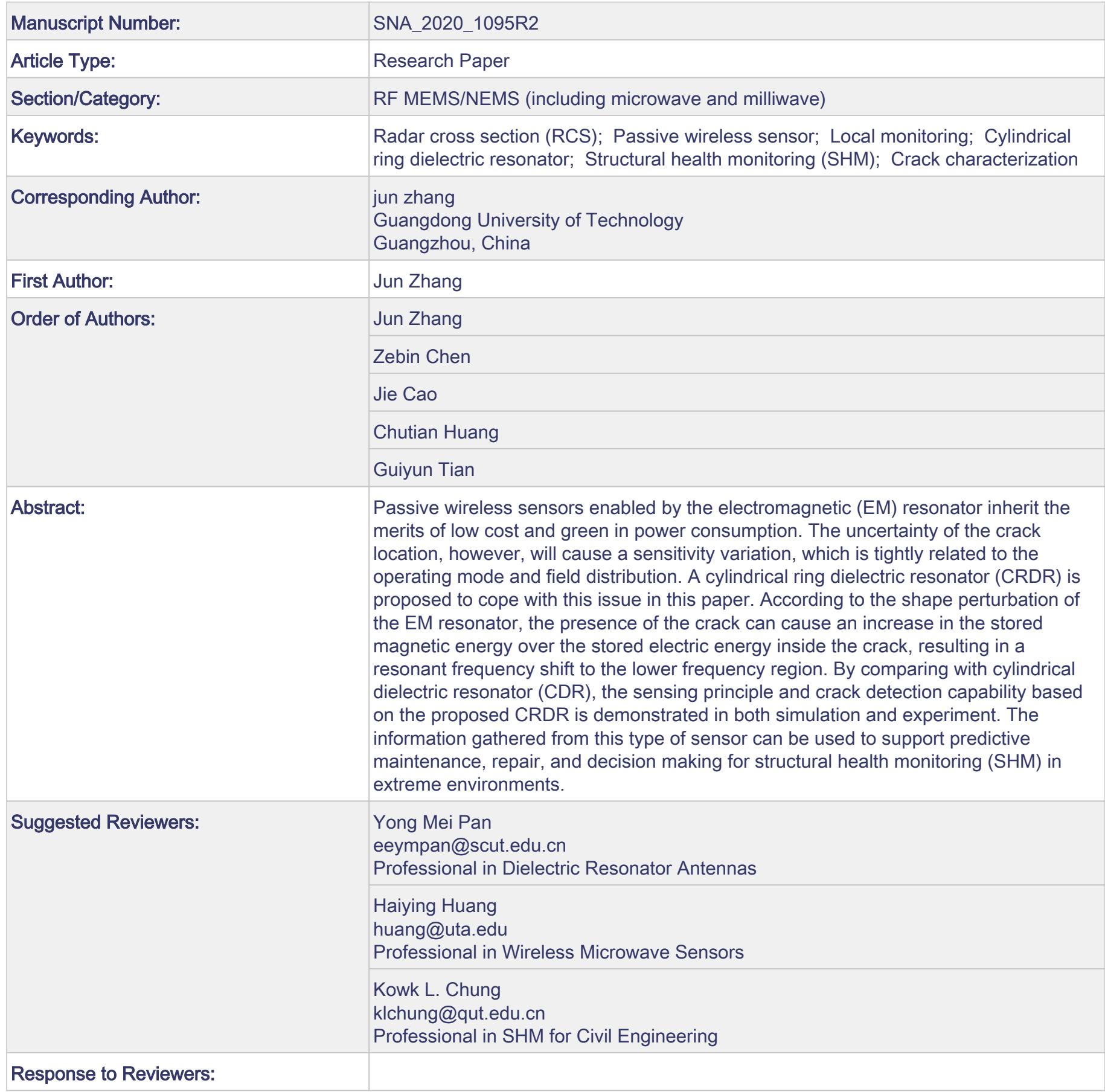




\section{Highlights}

- A cylindrical ring dielectric resonator for structural health monitoring in extreme environments

- The uncertainty of the crack tightly related to the operating mode and field distribution

- The shape perturbation causes an increase in the stored magnetic energy

- A uniform field in the center area to suppress the sensitivity variation 


\section{Abstract}

Passive wireless sensors enabled by the electromagnetic (EM) resonator inherit the merits of low cost and green in power consumption. The uncertainty of the crack location, however, will cause a sensitivity variation, which is tightly related to the operating mode and field distribution. A cylindrical ring dielectric resonator (CRDR) is proposed to cope with this issue in this paper. According to the shape perturbation of the EM resonator, the presence of the crack can cause an increase in the stored magnetic energy over the stored electric energy inside the crack, resulting in a resonant frequency shift to the lower frequency region. By comparing with cylindrical dielectric resonator (CDR), the sensing principle and crack detection capability based on the proposed CRDR is demonstrated in both simulation and experiment. The information gathered from this type of sensor can be used to support predictive maintenance, repair, and decision making for structural health monitoring (SHM) in extreme environments. 


\section{CRDR}

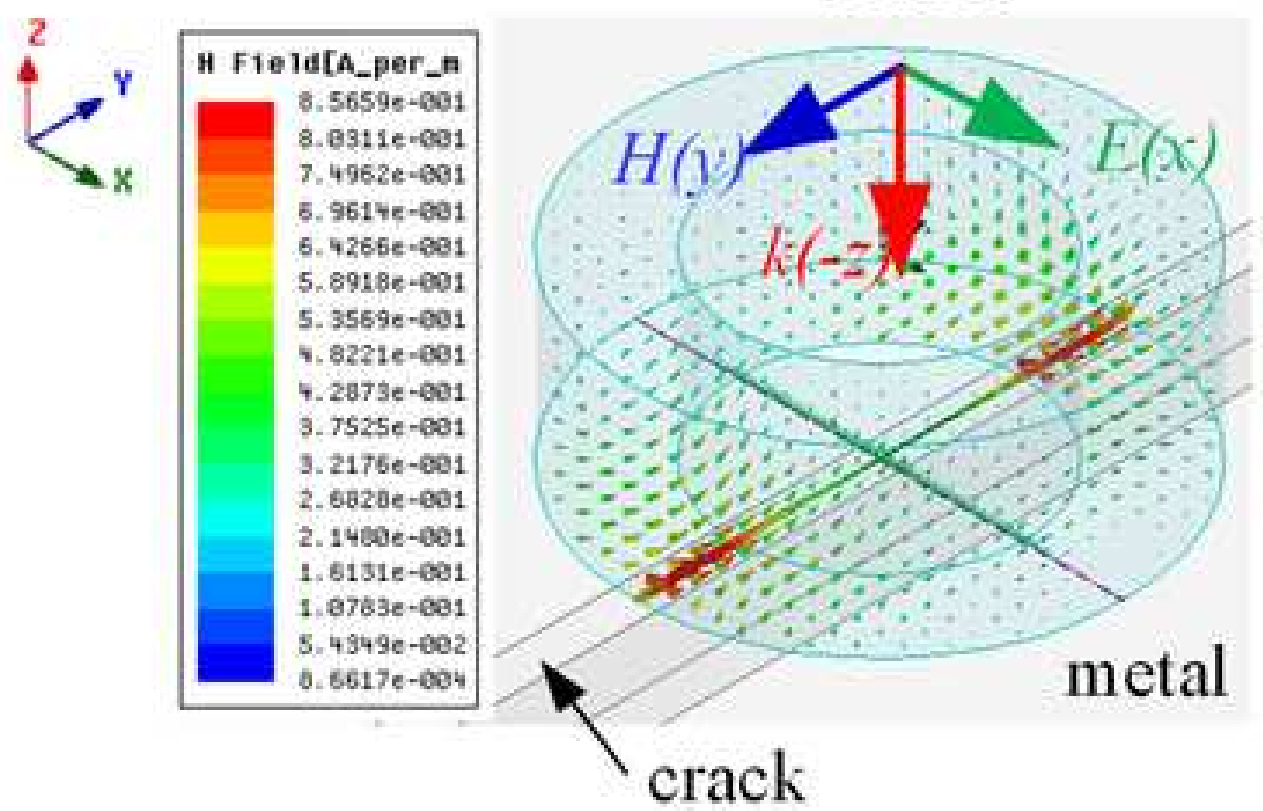

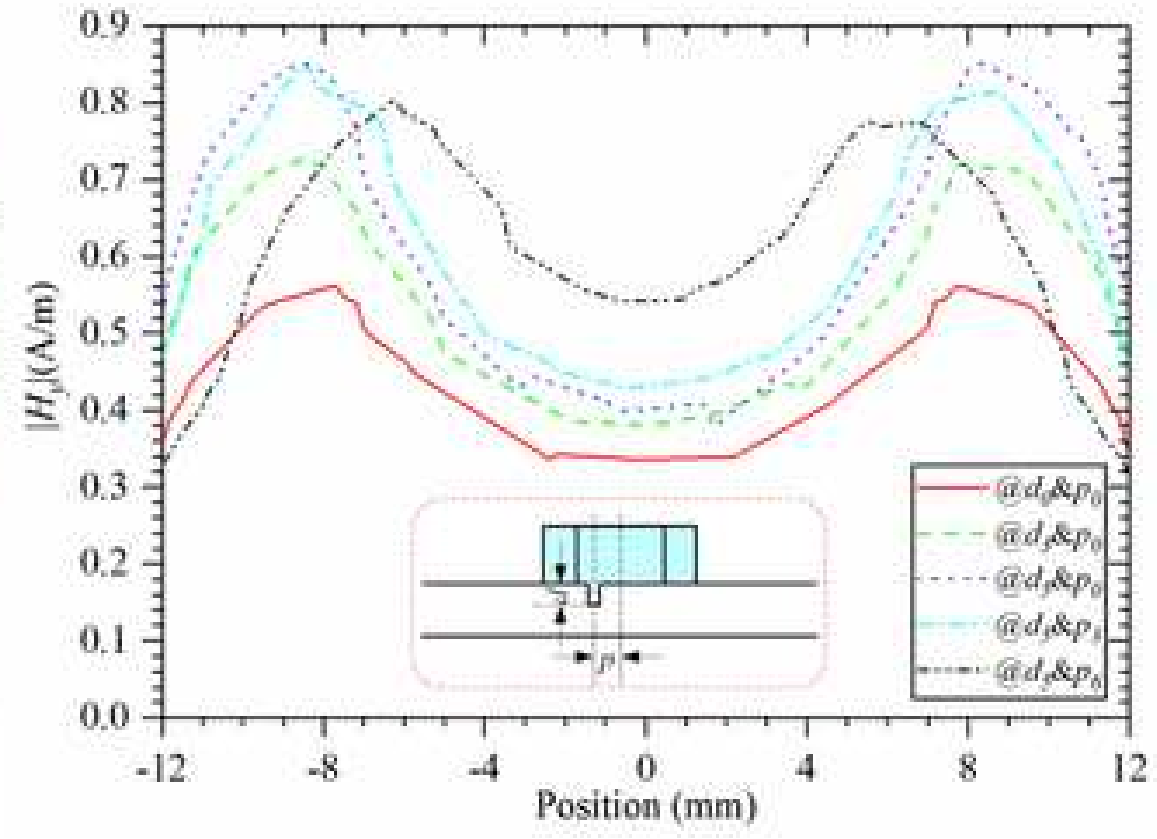




\title{
A Cylindrical Ring Dielectric Resonator based Passive Wireless Sensor for Position Insensitive Crack Monitoring
}

\author{
Jun Zhang ${ }^{1}$, Zebin Chen ${ }^{1}$, Jie Cao ${ }^{1}$, Chutian Huang ${ }^{1}$, and Guiyun Tian ${ }^{2,3}$
}

${ }^{1}$ School of Information Engineering, Guangdong University of Technology, Guangzhou 510006, China.
${ }^{2}$ School of Automation Engineering, University of Electronic Science and Technology of China, Chengdu 611731, China.
${ }^{3}$ School of Engineering, Newcastle University, Newcastle upon Tyne NE1 7RU, United Kingdom.
Corresponding author: Jun Zhang, e-mail: junzhang@gdut.edu.cn

\begin{abstract}
Passive wireless sensors enabled by the electromagnetic (EM) resonator inherit the merits of low cost and green in power consumption. The uncertainty of the crack location, however, will cause a sensitivity variation, which is tightly related to the operating mode and field distribution. A cylindrical ring dielectric resonator (CRDR) is proposed to cope with this issue in this paper. According to the shape perturbation of the EM resonator, the presence of the crack can cause an increase in the stored magnetic energy over the stored electric energy inside the crack, resulting in a resonant frequency shift to the lower frequency region. By comparing with cylindrical dielectric resonator (CDR), the sensing principle and crack detection capability based on the proposed CRDR is demonstrated in both simulation and experiment. The information gathered from this type of sensor can be used to support predictive maintenance, repair, and decision making for structural health monitoring (SHM) in extreme environments.
\end{abstract}

\section{Keywords}

Local monitoring, passive wireless sensor, crack characterization, cylindrical ring dielectric resonator, structural health monitoring (SHM), radar cross section (RCS)

\section{Introduction}

Cracks are common defects in civil engineering like bridges and railways. If these cracks are not detected in a timely manner, they can grow gradually and may result in a sudden failure of the component and catastrophic incidence [1]. The traditional structural health monitoring (SHM) methods use global responses such as vibration and local responses such as strains or a combination of both to assess the structure during in-service conditions or extreme climatic events [2]. Meanwhile, many non-destructive testing (NDT) techniques, e.g., ultrasonic [3], eddy current pulsed thermography [4], have been used for in-service integrity evaluation. The increase of the lift-off distance is one way to bridge the gap between NDT and SHM.

Antenna sensors are a passive and cost-effective way for locally monitoring defects immediately under the sensor [5]. The defect information can be wirelessly acquired and extracted from the radar cross section (RCS) directly or signal oriented for communication by radio frequency identification (RFID) technology. This type of local monitoring technique provides the possibility for tracking the progress of damage already identified in routine NDT inspection, or monitoring known hot-spots [6].

Owing to the defect induced impedance mismatching, the tradeoff in sensing and communication may limit the application of antenna sensors in the ultra-high frequency (UHF) band for chipped tags [7]. Recently, a chipless RFID sensor tag was proposed, integrating four tip-loaded dipole resonators as a 4-bit ID encoder and a circular microstrip patch antenna (CMPA) resonator as a crack sensor [8]. Due to free of electronic components, this type of sensor is applicable in extreme environments, such as aerospace engineering [9]. More recently, a complementary split-ring resonator (CSRR)-loaded array was used to simultaneously detect multiple cracks and localize the crack position [10].

A planar microwave resonator with a high-quality factor was proposed to detect coating breaches in industrial steel pipelines [11]. Recently, high-index dielectric resonators were intensively studied to manipulate the light with a significant reduction in energy dissipation and therefore an enhancement in device efficiency [12]. Inheriting from the field concentration into a compact structure, a cylindrical dielectric resonator (CDR) operated in the $\mathrm{HEM}_{11 \delta}$ mode can be naturally turned into a passive wireless sensor. The polarization of the CDR was configured for direction insensitive crack detection [13]. More recently, the arraying technique was used to detect crack of any orientation [14].

The communication capability of the chipless sensor, however, is constrained by the antenna profile as well as the loss of the substrate [15]. To make the sensor practical in engineering, the machine learning method can be used to enhance chipless RFID applications [16]. Luneburg lens was studied to enhance the RCS of the dielectric resonator [17]. Meanwhile, a circularly-polarized patch antenna was applied for multi-directional strain monitoring [18]. A thermally stable patch antenna was also designed for strain and crack sensing [19].

Because of the intrinsic resonant mode, the sensitivity of crack detection for an electromagnetic resonator is position sensitive [20], which hampers its practical applications in local monitoring. This paper is aiming to provide a solution in the mode induced sensitivity variation for crack detection and characterization based on the dielectric resonator. To this end, the shape perturbation method is studied to uncover the sensing principle of the dielectric resonators. A cylindrical ring dielectric resonator (CRDR) is proposed to generate a uniform field distribution to reduce the position induced sensitivity variation. The remaining part of this paper is organized as follows. Section II describes the structure and simulation setup. By comparing with the CDR, the sensing 
principle based on perturbation theory is analyzed in this section as well. The measurement setup and results are presented and discussed in Section III. Finally, Section IV draws the conclusion.

\section{Proposed sensor and its performance}

The CDR, which may operate in the transverse electric (TE), transverse magnetic (TM), or hybrid electromagnetic (HEM) mode, can be naturally turned into a passive wireless sensor for monitoring crack defects on metal. Owing to the boundary condition of the electric field, the $\mathrm{TE}_{01 \delta}$ mode cannot be excited when an electric wall is placed at the center of the symmetrical plane. The HEM $\mathrm{H}_{11 \delta}$ mode produces a broadside radiation pattern similar to that of a horizontal magnetic dipole whereas the TM $01 \delta$ mode gives an

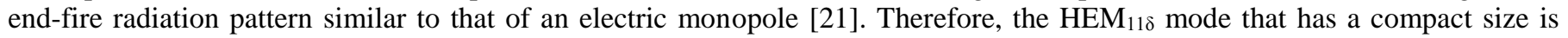
selected for the CDR and this mode can also be easily excited in the broadside direction.

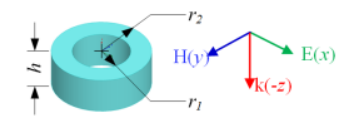

(a)
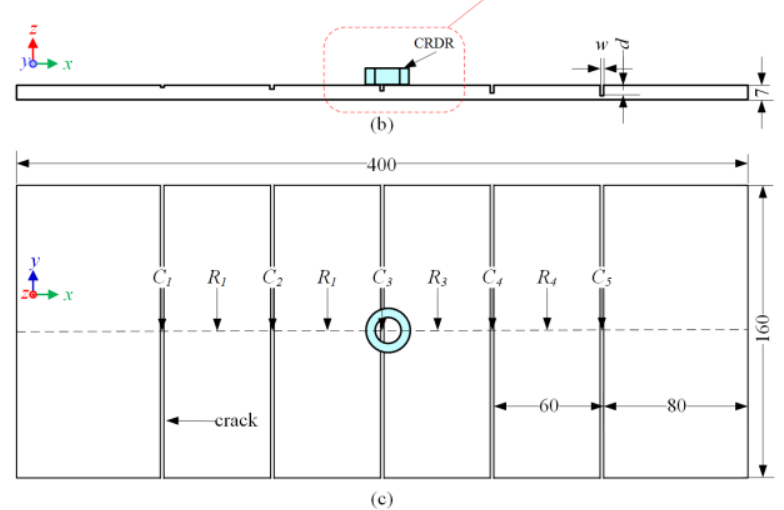

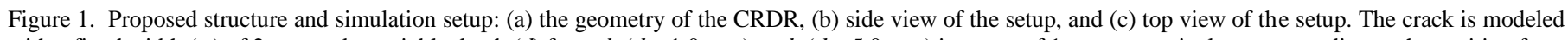

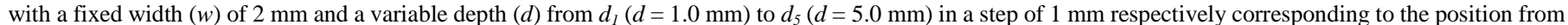

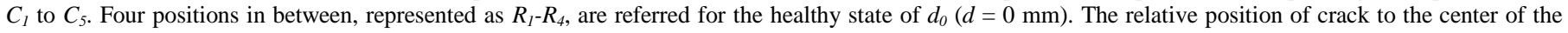

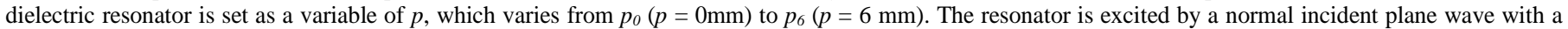
strength of the electric field ( $x$-oriented) of $1 \mathrm{~V} / \mathrm{m}$.
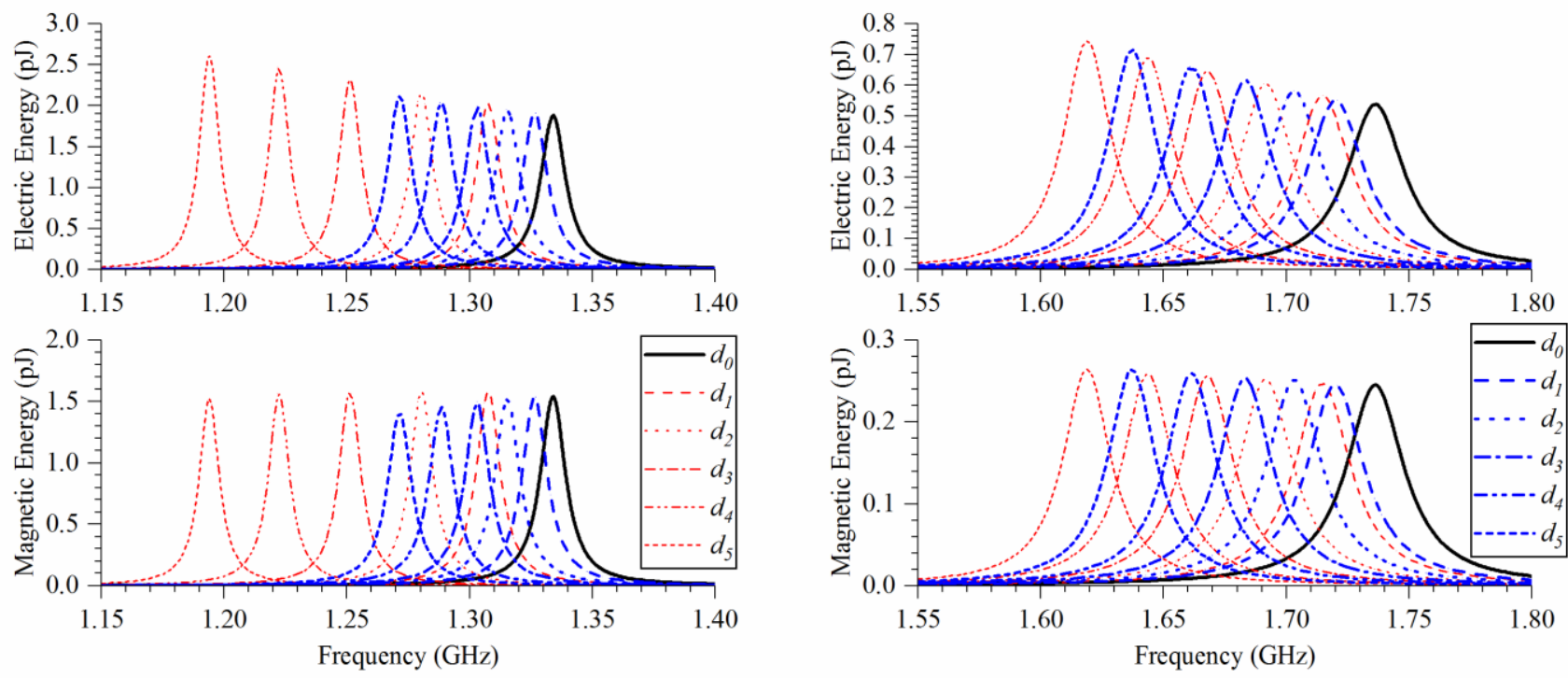

(a)

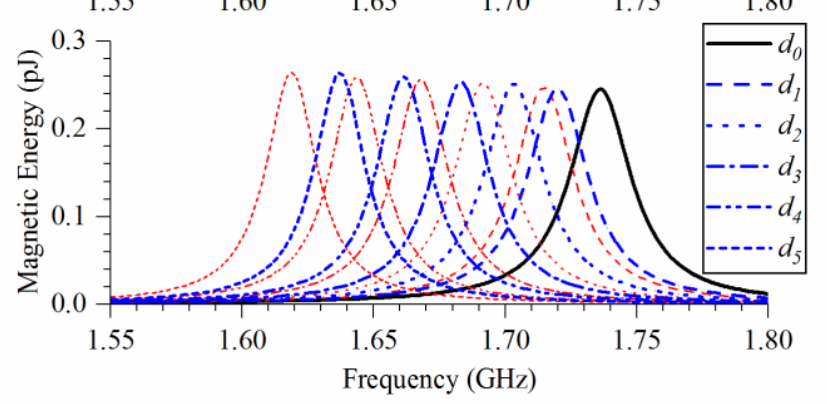

(b)

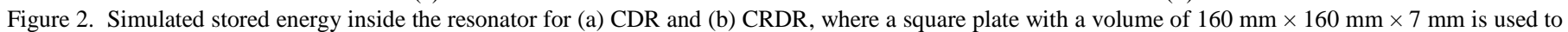

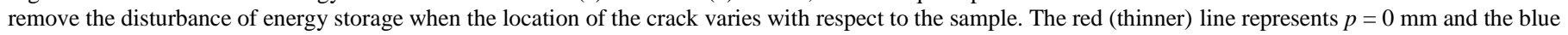
(thicker) line represents $p=6 \mathrm{~mm}$. The solid line represents the healthy state.

In this paper, a cylindrical ring dielectric resonator (CRDR) is proposed to suppress the position caused sensitivity variation, the structure of which is displayed in Figure 1(a). Ceramic material with a relative dielectric permittivity of 90 is used to achieve a compact size. For the CRDR, the inner radius $\left(r_{l}\right)$ is $7 \mathrm{~mm}$, the outer radius $\left(r_{2}\right)$ is $12 \mathrm{~mm}$, and the height $(h)$ is $9 \mathrm{~mm}$. The simulation setup is shown in Figures 1(b)-(c), where the position of the crack with respect to the dielectric resonator is set as a 
variable $(p)$. The overall size of the sample is $400 \mathrm{~mm} \times 160 \mathrm{~mm} \times 7 \mathrm{~mm}$. The material of the sample is aluminum, which is widely used in infrastructure. The dielectric resonator is directly mounted on the surface of the sample.

The CRDR supports the same type of mode as that of a CDR [22]. By removing the central part of a CDR, the resonant frequency of the CRDR will increase compared with the CDR [23]. The material perturbation method can be used to estimate the resonant frequency shift from the CDR to the CRDR [24]. To illustrate the energy perturbation inside the EM resonator, the stored magnetic and electric energy are displayed in Figure 2. Since the dielectric resonator operates in the $\mathrm{HEM}_{11 \delta}$ mode, we can find that the stored electric energy overwhelms the stored magnetic energy inside the resonator. Owing to the presence of the crack, the electric field pointed along the excitation direction (i.e., $x$-oriented in the configuration shown in Figure 1) appears. Therefore, there is an incremental increase in the stored electric energy when the crack increases or the position of crack moves from side to center of the resonator.

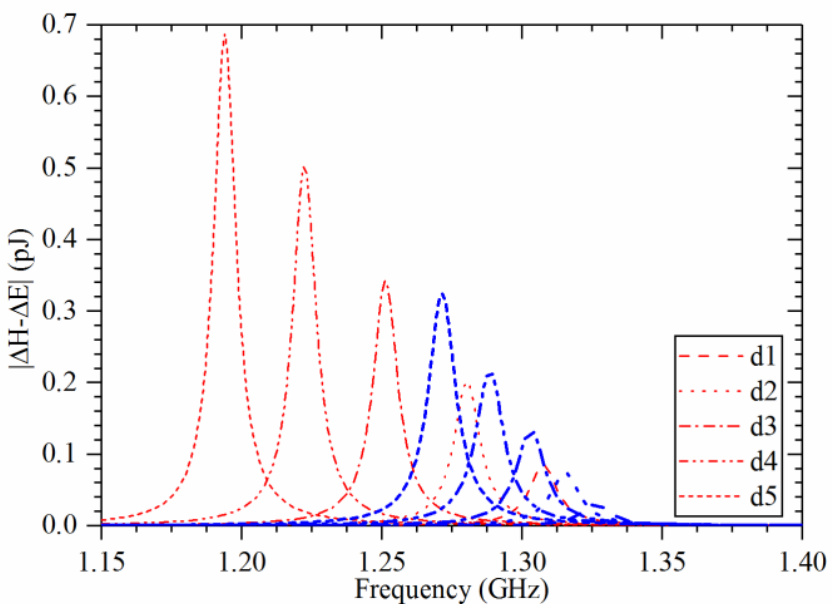

(a)

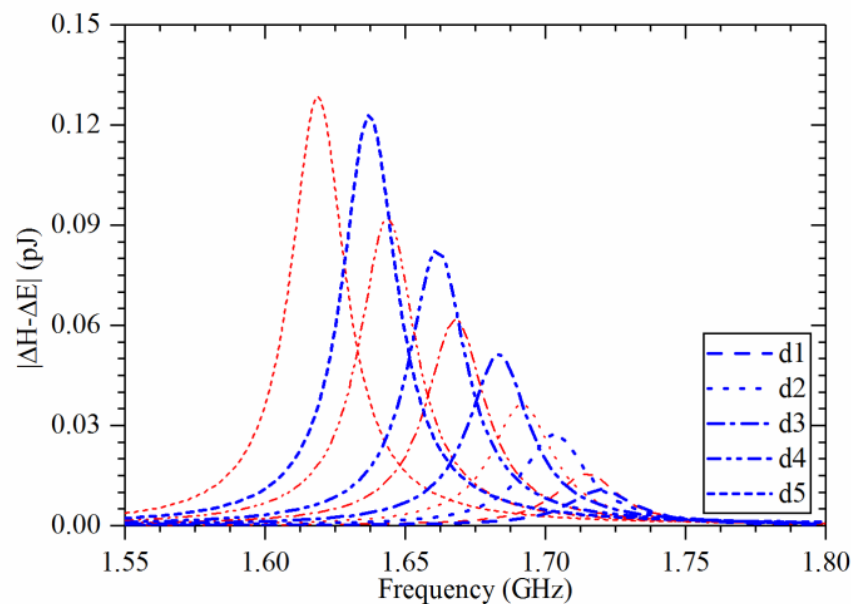

(b)

Figure 3. Simulated difference of the stored magnetic energy and the stored electric energy inside the crack for (a) CDR and (b) CRDR. The red (thinner) line represents $p=0 \mathrm{~mm}$ and the blue (thicker) line represents $p=6 \mathrm{~mm}$.

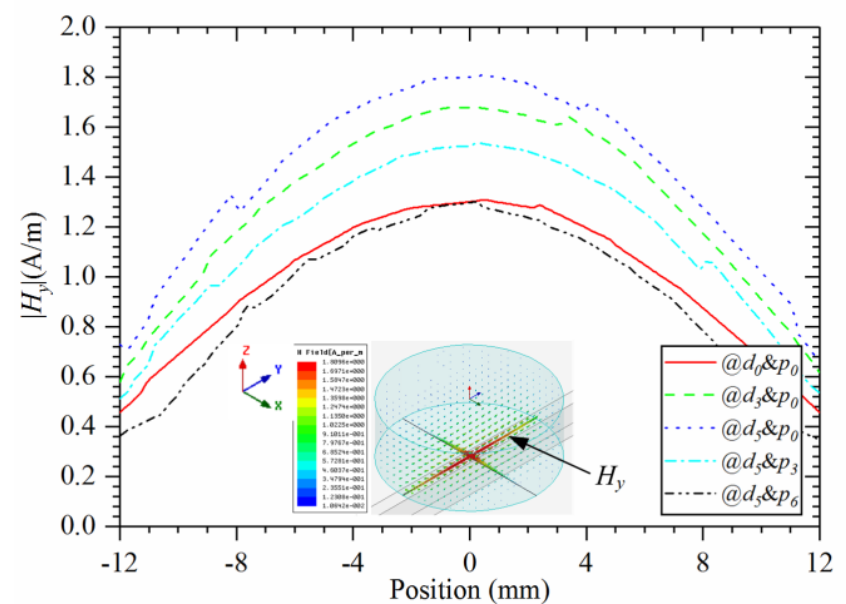

(a)

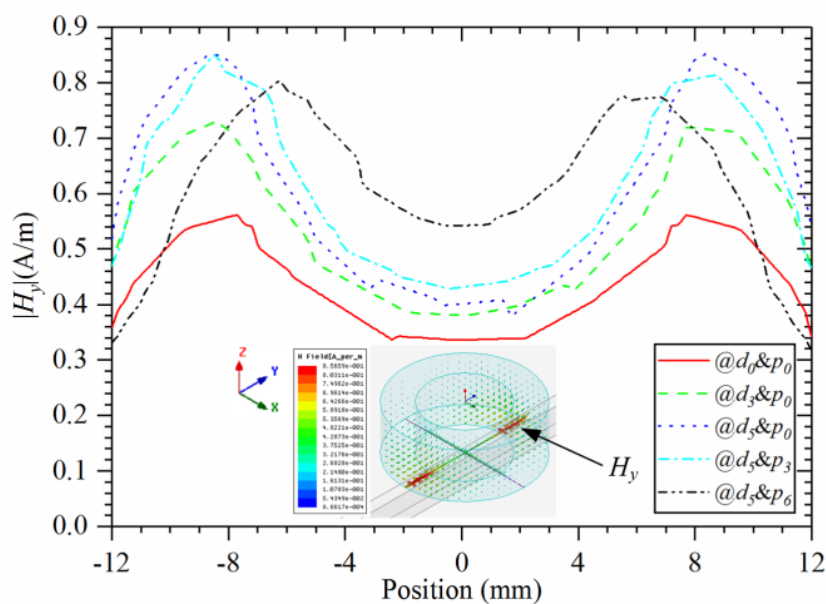

(b)

Figure 4. Extracted magnetic field of $x$-component along the crack at the resonant frequency (a) CDR and (b) CRDR, where a square plate with a volume of 160 mm $\times 160 \mathrm{~mm} \times 7 \mathrm{~mm}$ is used to remove the disturbance of energy storage when the location of the crack varies with respect to the plate.

From the perturbation theory, the resonant frequency would shift either up or down depending on whether there is an increase in the difference of the magnetic and electric energy inside the crack [24]. Similarly, the incident field in the microstrip line exciting a magnetic dipole in the slot can be found to excite a cylindrical dielectric resonator antenna [25]. Figure 3 illustrates the difference between the stored magnetic energy and the stored electric energy. Apparently, this difference will increase as the crack profile increases. The resonant frequency and the stored energy are changed from the original state to the perturbed one, the relationship can be written as [26]:

$$
\frac{\omega-\omega_{0}}{\omega_{0}}=\frac{\Delta W_{m}-\Delta W_{e}}{W_{m}+W_{e}} .
$$

Where $\omega$ and $\omega_{0}$ are the resonant frequency of the perturbed state and the original one, respectively. $\Delta W_{m}\left(\Delta W_{e}\right) \underline{\text { is the difference }}$ between the stored magnetic (electric) field energy of the original state (without crack) and that of the perturbed state (with crack). 
$W_{m}\left(W_{e}\right)$ is the magnetic (electric) energy stored in the resonator at the original state. Nevertheless, the perturbation in the magnetic (electric) field is tightly related to the position of crack with respect to the field distribution of the dielectric resonator [27]. The influence of the irregular crack, for example, zigzag shape, can be expected and predicted using the shape perturbation theory as well.

To understand the field distribution of the CDR and CRDR further, Figure 4 shows the magnetic field of the dielectric resonator on the metallic surface. Comparing the CRDR with the CDR, both the field concentration and the Q-factor are reduced [28]. The field in the CRDR is, therefore, smoother. The perturbation due to the presence of the crack on metal may be more uniform.

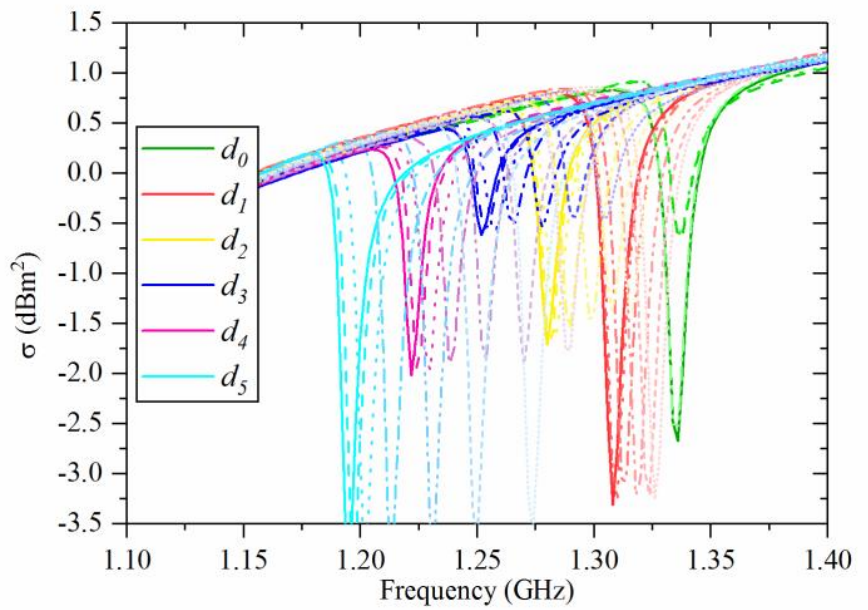

(a)

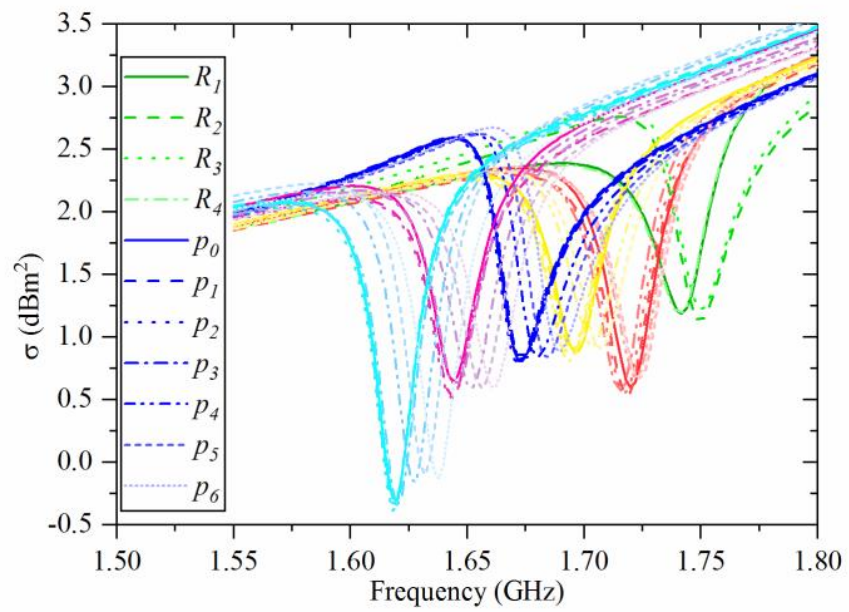

(b)

Figure 5. Simulated radar cross section for (a) CDR and (b) CRDR, where the simulation setup is shown in Figure 1(c). (a) and (b) share the same legend.

The simulated RCS for both the CDR and CRDR is shown in Figure 5. This radar echo plays an indicator of the communication capability of the resonator. Owing to the Mie-type resonances [29], the radiation damping yields a dip in the scattering response of the localized CDR and CRDR on metal. The ground plane size, however, has a slight influence on the resonant frequency of the resonator. Regardless of the crack depth, the position of the dielectric resonator with respect to the sample has an apparent influence on the amplitude of the RCS.

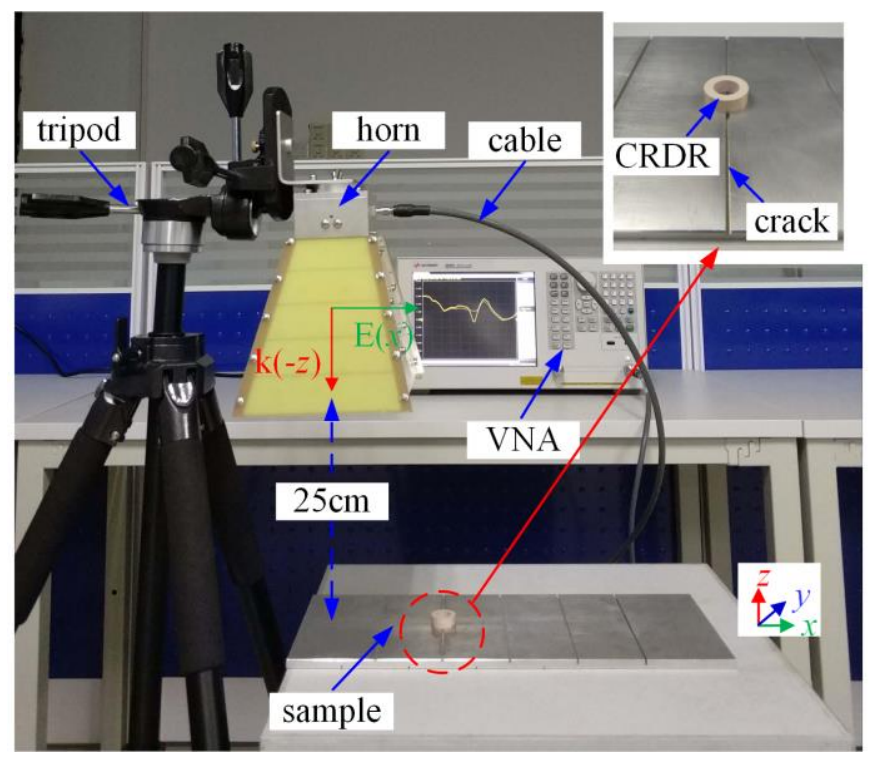

Figure 6. Measurement setup.

\section{Measurement and results}

The experimental setup in the lab is displayed in Figure 6. A well-matched horn antenna operated in 1-18GHz was used to interrogate with the dielectric resonator. The reflection coefficient $\left(S_{11}\right)$ of the horn antenna was measured by a vector network analyzer (VNA) of E5071C from Keysight Technologies. To simplify the instrumentation, the RCS can be extracted from the S11 of a well-matched horn antenna [30]. The configuration is the same with [13], where the $\Delta\left|\mathrm{S}_{11}\right|$ was used as a sensing variable, 
which can partly remove the echoes from the sample and nearby objects. The resonant frequency from the dielectric resonator [31] was exacted from the valley of the $\Delta\left|\mathrm{S}_{11}\right|$.

An aluminum alloy 6061 sized of $400 \mathrm{~mm} \times 160 \mathrm{~mm} \times 7 \mathrm{~mm}$ was sampled. The detail size of the sample is the same as the simulation setup shown in Figures 1(b)-(c). The responses in the four positions $\left(R_{1}-R_{4}\right)$ were recorded, referring to the healthy state. The polarization ( $E$-field) of the horn antenna was configured to be orthogonal with the crack direction to maximize the sensitivity. According to the shape perturbation theory, the sensitivity is tightly related to crack width; therefore, the sensitivity in this paper is defined as $S=\Delta f /\left(w^{*} d\right)$.

The simulated and measured resonant frequencies under variations of crack position and crack depth are shown in Figure 7. For the CDR at the four reference positions, the simulated resonant frequency varies from $1.336 \mathrm{GHz}$ to $1.336 \mathrm{GHz}$ with an average value of $1.336 \mathrm{GHz}$. When $d=5 \mathrm{~mm}$, the simulated resonant frequency varies from $1.194 \mathrm{GHz}$ at $p_{0}$ to $1.274 \mathrm{GHz}$ at $p_{6}$ with an average value of $1.223 \mathrm{GHz}$. Therefore, the simulated sensitivity of $S$ is $-14.2 \mathrm{MHz} / \mathrm{mm}^{2}$ at $p_{0}$ and $-6.2 \mathrm{MHz} / \mathrm{mm}^{2}$ at $p_{6} \mathrm{~mm}$. The measured resonant frequency varies from $1.320 \mathrm{GHz}$ to $1.328 \mathrm{GHz}$ with an average value of $1.323 \mathrm{GHz}$ at the four reference positions. When $d=5 \mathrm{~mm}$, the measured resonant frequency varies from $1.219 \mathrm{GHz}$ at $p_{0}$ to $1.264 \mathrm{GHz}$ at $p_{6}$ with an average value of $1.235 \mathrm{GHz}$. Therefore, the measured sensitivity of $S$ is $-10.4 \mathrm{MHz} / \mathrm{mm}^{2}$ at $p_{0}$ and $-5.9 \mathrm{MHz} / \mathrm{mm}^{2}$ at $p_{6}$.

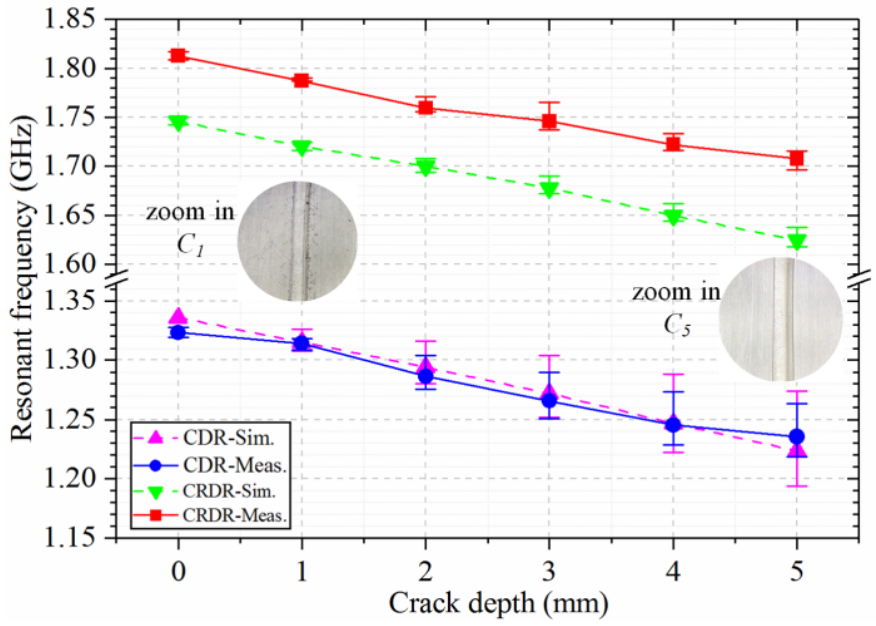

Figure 7. Measured resonant frequency under variations of crack position and depth.

For the CRDR at the four reference positions, the simulated resonant frequency varies from $1.742 \mathrm{GHz}$ to $1.750 \mathrm{GHz}$ with an average value of $1.746 \mathrm{GHz}$. When $d=5 \mathrm{~mm}$, the simulated resonant frequency varies from $1.618 \mathrm{GHz}$ at $p_{0}$ to $1.638 \mathrm{GHz}$ at $p_{6} \mathrm{~mm}$ with an average value of $1.625 \mathrm{GHz}$. Therefore, the simulated sensitivity of $S$ is $-12.8 \mathrm{MHz} / \mathrm{mm}^{2}$ at $p_{0}$ and $-10.8 \mathrm{MHz} / \mathrm{mm}^{2}$ at $p_{6}$. The measured resonant frequency varies from $1.809 \mathrm{GHz}$ to $1.817 \mathrm{GHz}$ with an average value of $1.812 \mathrm{GHz}$ at the four reference positions. When $d=5 \mathrm{~mm}$, the measured resonant frequency varies from $1.697 \mathrm{GHz}$ at $p_{0}$ to $1.716 \mathrm{GHz}$ at $p_{6}$ with an average value of $1.708 \mathrm{GHz}$. Therefore, the measured sensitivity of $S$ is $-11.5 \mathrm{MHz} / \mathrm{mm}^{2}$ at $p_{0}$ and $-9.6 \mathrm{MHz} / \mathrm{mm}^{2}$ at $p_{6}$. However, there is a slight discrepancy in the simulated and measured resonant frequency of the CRDR at the healthy state, which is attributed to the tolerance of the dielectric constant of the ceramic material.

Compared with the CDR, the sensitivity of the CRDR is expected to be decreased when the field concentration is reduced. The reduction of the sensitivity, however, can be partly compensated by the increase of the operating frequency while the same ceramic material is used. At the range of $p_{0}$ to $p_{6}$ when the crack depth varies from $0 \mathrm{~mm}$ to $5 \mathrm{~mm}$, the simulated average sensitivity of $S$ is $-11.3 \mathrm{MHz} / \mathrm{mm}^{2}$ for the CDR and $-12.1 \mathrm{MHz} / \mathrm{mm}^{2}$ for the CRDR. The measured average sensitivity of $S$ is $-8.8 \mathrm{MHz} / \mathrm{mm}^{2} \mathrm{for}$ the CDR and $-10.4 \mathrm{MHz} / \mathrm{mm}^{2}$ for the CRDR. Since the air gap between the metal and dielectric resonator will induce a significant shift of the resonant frequency to the higher frequency region, an obvious abnormality in the position of $C_{5}$ can be observed due to the surface elevation. For both the CDR and CRDR, the measured maximum sensitivities are lower than the simulated ones, which are attributed to the measurement error in the polarization and fabrication error in the crack size.

Above all, the extent of the uncertainty caused by the crack position variation can be suppressed by the proposed CRDR when compared with its counterpart of the CDR. The error bars become large when the position of the crack moves outside of the extended range, e.g., when $p$ is large than $6 \mathrm{~mm}$ for the CRDR.

\section{Conclusion}

In this paper, a compact dielectric resonator-based wireless passive sensor has been explored for crack monitoring in practical application. The sensing principle is analyzed in terms of perturbation in energy storage. We can find that the increase in the difference of the stored magnetic energy and the electric energy causes a decrease in the resonant frequency, which is tightly related to the field distribution with respect to crack location. Compared with the previous structure of the CDR, the proposed CRDR has a comparable sensitivity but a more uniform magnetic field distribution. Consequently, more robustness in crack 
detection can be expected. The proposed cell may provide possibilities for array design such as dielectric analog meta-surfaces [32]. Owing to free of electronic devices, this type of sensor can be permanently installed for local monitoring in extreme environments.

\section{Declaration of Conflicting Interests}

The author(s) declared no potential conflicts of interest with respect to the research, authorship, and/or publication of this article.

\section{Funding}

This work was supported in part by the National Natural Science Foundation of China (NSFC) [61701120 and 61527803] and in part by the Guangdong Basic and Applied Basic Research Foundation [2020A1515010723].

\section{References}

[1] F. Mazlumi, S. H. H. Sadeghi, and R. Moini, "Interaction of an open-ended rectangular waveguide probe with an arbitrary-shape surface crack in a lossy conductor," IEEE Transactions on Microwave Theory and Techniques, vol. 54, pp. 3706-3711, Oct. 2006.

[2] S. Sony, S. Laventure, and A. Sadhu, "A literature review of next-generation smart sensing technology in structural health monitoring," Structural Control \& Health Monitoring, vol. 26, Mar. 2019.

[3] N. Yacef, T. Bouden, and M. Grimes, "Accurate ultrasonic measurement technique for crack sizing using envelope detection and differential evolution," NDT \& E International, vol. 102, pp. 161-168, Mar. 2019.

[4] Y. Z. Wang, B. Gao, W. L. Woo, G. Y. Tian, X. Maldague, L. Zheng, et al., "Thermal Pattern Contrast Diagnostic of Microcracks With Induction Thermography for Aircraft Braking Components," IEEE Transactions on Industrial Informatics, vol. 14, pp. 5563-5574, Dec. 2018.

[5] I. Mohammad and H. Huang, "Monitoring fatigue crack growth and opening using antenna sensors," Smart Materials and Structures, vol. 19, May 2010.

[6] P. Cawley, "Structural health monitoring: Closing the gap between research and industrial deployment," Structural Health Monitoring, vol. 17, pp. $1225-1244,2018$.

[7] J. Zhang, G. Y. Tian, A. M. J. Marindra, A. I. Sunny, and A. B. Zhao, "A Review of Passive RFID Tag Antenna-Based Sensors and Systems for Structural Health Monitoring Applications," Sensors, vol. 17, Feb. 2017.

[8] A. M. J. Marindra and G. Y. Tian, "Chipless RFID Sensor Tag for Metal Crack Detection and Characterization," IEEE Transactions on Microwave Theory and Techniques, vol. 66, pp. 2452-2462, May 2018.

[9] J. M. Boccard, T. Aftab, J. Hoppe, A. Yousaf, R. Hutter, and L. M. Reindl, "High-Resolution, Far-Field, and Passive Temperature Sensing up to 700 degrees C Using an Isolated ZST Microwave Dielectric Resonator," IEEE Sensors Journal, vol. 16, pp. 715-722, Feb. 2016.

[10] A. Salim, A. H. Naqvi, A. D. Pham, and S. Lim, "Complementary Split-Ring Resonator (CSRR)-Loaded Sensor Array to Detect Multiple Cracks: Shapes, Sizes, and Positions on Metallic Surface," IEEE Access, vol. 8, pp. 151804-151816, 2020.

[11] M. H. Zarifi, S. Deif, M. Abdolrazzaghi, B. Chen, D. Ramsawak, M. Amyotte, et al., "A Microwave Ring Resonator Sensor for Early Detection of Breaches in Pipeline Coatings," IEEE Transactions on Industrial Electronics, vol. 65, pp. 1626-1635, Feb. 2018.

[12] S. Jahani and Z. Jacob, "All-dielectric metamaterials," Nature Nanotechnology, vol. 11, pp. 23-36, Jan. 2016.

[13] J. Zhang, H. X. Huang, C. T. Huang, B. S. Zhang, Y. Li, K. Wang, et al., "A Configurable Dielectric Resonator-Based Passive Wireless Sensor for Crack Monitoring," IEEE Transactions on Antennas and Propagation, vol. 67, pp. 5746-5749, Aug. 2019.

[14] S. Dey, R. Bhattacharyya, S. E. Sarma, and N. C. Karmakar, "A Novel "Smart Skin" Sensor for Chipless RFID Based Structural Health Monitoring Applications," IEEE Internet of Things Journal, pp. 1-1, 2020.

[15] J. Zhang, B. Huang, G. Zhang, and G. Y. Tian, "Wireless Passive Ultra High Frequency RFID Antenna Sensor for Surface Crack Monitoring and Quantitative Analysis," Sensors, vol. 18, Jul. 2018.

[16] S. Jeong, J. G. D. Hester, W. J. Su, and M. M. Tentzeris, "Read/Interrogation Enhancement of Chipless RFIDs Using Machine Learning Techniques," IEEE Antennas and Wireless Propagation Letters, vol. 18, pp. 2272-2276, Nov 2019.

[17] A. A. Abbas, M. El-Absi, A. Abuelhaija, K. Solbach, and T. Kaiser, "High RCS Passive Tag based on Dielectric Resonator-2D Lens Combination," 2019 12th German Microwave Conference (Gemic), pp. 5-8, 2019.

[18] K. L. Chung, L. L. Wang, J. L. Luo, Y. S. Li, and Y. S. Li, "Comparative study on directional sensitivity of patch-antenna-based strain sensors," International Journal of Rf and Microwave Computer-Aided Engineering, vol. 30, Nov. 2020.

[19] D. Li and Y. Wang, "Thermally Stable Wireless Patch Antenna Sensor for Strain and Crack Sensing," Sensors, vol. 20, Jul. 2020.

[20] L. Ke, Z. P. Liu, and H. J. Yu, "Characterization of a Patch Antenna Sensor's Resonant Frequency Response in Identifying the Notch-Shaped Cracks on Metal Structure," Sensors, vol. 19, Jan. 2019.

[21] S. M. Shum and K. M. Luk, "Stacked annular ring dielectric resonator antenna excited by axi-symmetric coaxial probe," IEEE Transactions on Antennas and Propagation, vol. 43, pp. 889-892, Aug. 1995.

[22] R. K. Mongia and P. Bhartia, "Dielectric resonator antennas-A review and general design relations for resonant frequency and bandwidth," International Journal of Microwave and Millimeter - Wave Computer - Aided Engineering, vol. 4, pp. 230-247, 1994.

[23] U. M. Khan and M. Milutinovic, "Dual polarized dielectric resonator antennas," Master, Chalmers University of Technology 2010.

[24] B.-T. Lim, "Dielectric resonator antennas: Theory and design," Master, Massachusetts Institute of Technology, 1999.

[25] R. K. Mongia, A. Ittipiboon, Y. M. M. Antar, P. Bhartia, and M. Cuhaci, "A half-split cylindrical dielectric resonator antenna using slot-coupling," IEEE Microwave and Guided Wave Letters, vol. 3, pp. 38-39, 1993.

[26] J. Zhang, J. Cao, Y. Li, C. Huang, and K. Wang, "A Quantitative Study of Crack Monitoring for Electromagnetic Resonators by Perturbation Method," presented at the 3rd International Conference on Electronic Information and Communication Technology, Shenzhen, China, 2020.

[27] J. A. Kong, Electromagnetic Wave Theory: EMW Publishing, 2000.

[28] M. V. Rybin, K. L. Koshelev, Z. F. Sadrieva, K. B. Samusev, A. A. Bogdanov, M. F. Limonov, et al., "High-Q Supercavity Modes in Subwavelength Dielectric Resonators," Physical Review Letters, vol. 119, Dec. 2017.

[29] K. Koshelev, G. Favraud, A. Bogdanov, Y. Kivshar, and A. Fratalocchi, "Nonradiating photonics with resonant dielectric nanostructures," Nanophotonics, vol. 8, pp. 725-745, May 2019.

[30] P. V. Nikitin and K. V. S. Rao, "Theory and measurement of backscattering from RFID tags," IEEE Antennas and Propagation Magazine, vol. 48, pp. 212-218, Dec. 2006.

[31] A. A. Abbas, M. El-Absi, A. Abuelhaija, K. Solbach, and T. Kaiser, "Metallic reflectors with notched RCS spectral signature using dielectric resonators," Electronics Letters, vol. 56, pp. 273-276, Mar. 2020.

[32] H. H. Hsiao, C. H. Chu, and D. P. Tsai, "Fundamentals and Applications of Metasurfaces," Small Methods, vol. 1, Apr. 2017. 



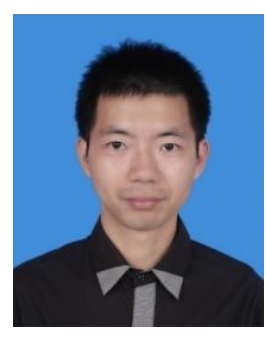

Jun Zhang received the B.Eng. degree in information engineering from the South China University of Technology, Guangzhou, China, in 2008 and the Ph.D. degree in radio physics from the Sun Yatsen University, Guangzhou, China, in 2013.

He is currently an Associate Professor with the School of Information Engineering, Guangdong University of Technology, Guangzhou, China. His research interests include electromagnetic theory and inverse problem, 5G antennas, passive wireless sensors, and structural health monitoring (SHM).

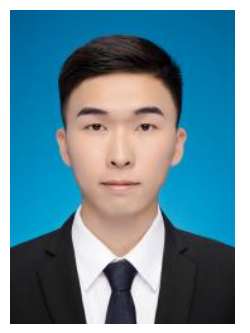

Zebin Chen received the B.Eng. degree in information engineering from Guangdong University of Technology, Guangzhou, China, in 2020.

$\mathrm{He}$ is currently working toward the M.S. degree in information and communication engineering, Guangdong University of Technology, Guangzhou, China. His research interests include electromagnetic theory, millimeter wave antenna, and passive wireless sensors.

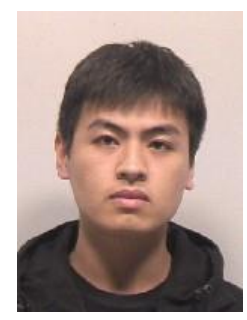

Jie Cao received the B.Eng. degree in Communication Engineering from Wanjiang University of Technology, Maanshan, China, in 2019.

He is currently working toward the M.S. degree in integrated circuit engineering, Guangdong University of Technology, Guangzhou, China. His current research interests include antenna array and dielectric resonators.

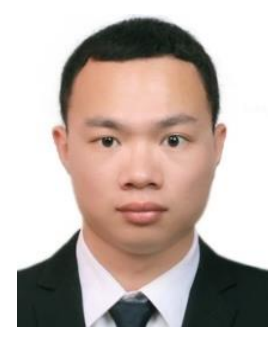

Chutian Huang received the B.Eng. degree in information engineering from Guangdong University of Technology, Guangzhou, China, in 2018.

He is currently working toward the M.S. degree in information and communication engineering, Guangdong University of Technology, Guangzhou, China. His research interests include electromagnetic theory, artificial electromagnetic materials and devices, and passive wireless sensors.

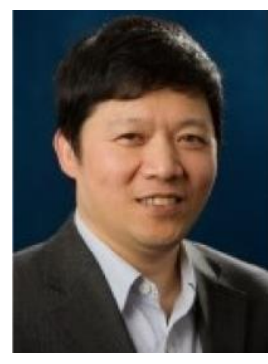

Guiyun Tian received the B.Sc. degree in metrology and instrumentation and the M.Sc. degree in precision engineering from the University of Sichuan, Chengdu, China in 1985 and 1988, respectively, and the Ph.D. degree from the University of Derby, Derby, U.K., in 1998.

$\mathrm{He}$ is currently a Chair Professor in sensor technologies with the School of Engineering in the Newcastle University, Newcastle upon Tyne, U.K. He is also an adjunct professor with the School of Automation Engineering, University of Electronic Science and Technology of China, Chengdu, China. His main interests include electromagnetic sensors, sensor array and networks, non-destructive testing \& evaluation, advanced signal processing and integrative systems. He has coordinated several research projects from the Engineering and Physical Sciences research Council (EPSRC), Royal Academy of Engineering and FP7. Also he has good collaborative links with leading industrial companies such as Airbus, Rolls Royce, BP, nPower, Network Rail and TWI among others. 


\section{Declaration of interests}

$\bigotimes$ The authors declare that they have no known competing financial interests or personal relationships that could have appeared to influence the work reported in this paper.

$\square$ The authors declare the following financial interests/personal relationships which may be considered as potential competing interests:

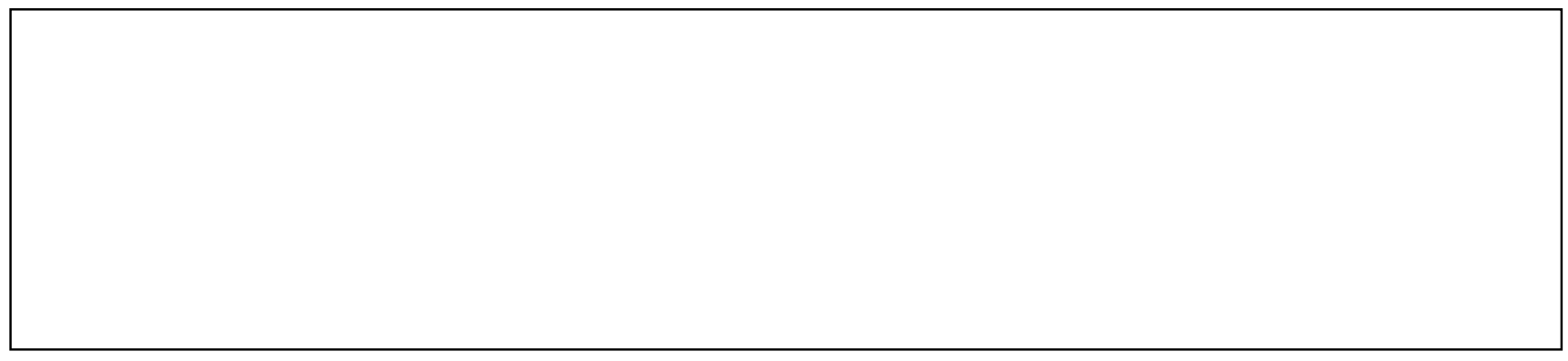


Jun Zhang: Conceptualization, Methodology.

Zebin Chen: Investigation, Validation, Writing- Original draft preparation.

Jie Cao: Software, Data curation.

Chutian Huang: Writing- Reviewing and Editing.

Guiyun Tian: Supervision. 
Click here to access/download Supplementary Material ICEICT2020_Accepted.pdf 